\title{
Módulos elásticos isotrópicos em sedimentos turbidíticos da Bacia de Campos
} Lidiane de Souza Garcia \& Jorge Leonardo Martins, COGE, Observatório Nacional, MCT

Copyright 2010, SBGf - Sociedade Brasileira de Geofísica. Este texto foi preparado para a apresentação no IV Simpósio Brasileiro de Geofísica, Brasília, 14 a 17 de novembro de 2010. Seu conteúdo foi revisado pelo Comitê Técnico do IV SimBGf, mas não necessariamente representa a opinião da SBGf ou de seus associados. É proibida a reprodução total ou parcial deste material para propósitos comerciais sem prévia autorização da SBGf.

\section{RESUMO}

A avaliação dos módulos elásticos de um pacote sedimentar é de grande importância para a engenharia de perfuração, pois contribui para a minimização dos riscos inerentes às operações de perfuração. A investigação da variação das constantes elásticas de rochas sedimentares é possível a partir de perfis geofísicos de poços. Nesse trabalho, utilizamos perfis geofísicos de um poço da região central do reservatório de Namorado, bacia de Campos, para construir perfis representativos da variação de módulos elásticos isotrópicos em profundidade. Os resultados mostram que as magnitudes das estimativas dos módulos elásticos estão dentro dos intervalos esperados para o ambiente sedimentar investigado. Aplicaremos a mesma metodologia usando outros perfis geofísicos em poços adjacentes que atravessam o mesmo intervalo sedimentar, a fim de construir mapas da variação destes módulos elásticos na formação geológica que contém o reservatório Namorado.

\section{INTRODUÇÃO}

A avaliação dos módulos elásticos constitui uma etapa crucial na obtenção de informações adicionais para o planejamento e execução do programa de perfuração de poços em reservatórios de petróleo e gás. Destaca-se o uso dessas informações na minimização dos riscos de perfuração, conduzindo à maximização dos custos no desenvolvimento do campo petrolífero (Dewan, 1983; Elis, 1987).

Para avaliação dos módulos elásticos, torna-se necessário o uso de perfis de densidade efetiva (RHOB), e sônicos de ondas P e S (DTCO e DTSM), respectivamente. No presente trabalho, apresentamos parte dos resultados de um projeto de dissertação de mestrado que tem, por objetivo inicial, avaliar as propriedades elásticas isotrópicas e, posteriormente, anisotrópicas, do reservatório Namorado, inserido na formação Macaé superior, bacia de Campos. Trata-se de uma típica bacia de margem divergente, tendo sua origem relacionada ao rompimento do supercontinente Gondwana e conse- qüente formação do Oceano Atântico. Nesta bacia, a interligação das rochas geradoras e das rochas reservatórios pós-evaporíticas está associada à tectônica profunda (fase rift) e à movimentação adiastrófica (halocinese). A migração de hidrocarbonetos é facilitada pela criação de "janelas"nos evaporitos (Trigre e Lucchesi, 1986).

No intervalo sedimentar do reservatório Namorado, estimamos as seguintes propriedades elásticas: as constantes de Lamé ( $\lambda$ e $\mu$ ), o módulo de compressão ou incompressibilidade $(\kappa)$, o módulo de Young $(E)$ e a razão de Poisson $(\sigma)$. Efetuamos as estimativas a partir do uso dos perfis geofísicos do conjunto de dados denominado "Campo Escola Namorado", cedido pela Agência Nacional do Petróleo, Gás e Biocombustível às instituições de ensino e pesquisa para fins didáticos. Perfis geofísicos fundamentais de 39 poços contidos nesse conjunto de dados registram propriedades físicas de rochas sedimentares turbidíticas que formam o reservatório Namorado. Todos os poços possuem registros dos perfis de densidade, e apenas cinco poços possuem perfis sônicos de onda P. A tarefa de estimar estes perfis ausentes para esse conjunto de dados foi executada nos trabalhos de Augusto \& Martins (2008) e Augusto (2009) a partir do uso de modelos empíricos. O conjunto de dados em questão são das décadas de 70-80 e não possuem os valores de perfil sônico de onda S. Para contornar a ausência de informação de velocidade de onda $S$, usamos a metodologia proposta em Lee (2006). Usando as informações de densidade e de velocidade de ondas $P$ e S, construímos perfis representativos da variação dos parâmetros elásticos no intervalo sedimentar sob investigação. Perfis compostos de alguns poços auxiliaram na correlação da variação dos módulos elásticos com as facies geológicas correspondentes.

\section{METODOLOGIA}

Para construir perfis de módulos elásticos usando os dados de perfilagem do "Campo Escola Namorado", aplicamos os seguintes procedimentos: (1) estimativa dos perfis de velocidade de ondas compressionais ausentes, usando as relações empíricas em Augusto (2009), Castagna et al. (1985), Han et al. (1986) e Miller \& Stewart (1990); (2) estimativa dos perfis de velocidade de ondas cisalhantes, usando a metodologia proposta em Lee (2006) e; (3) estimativa dos módulos elásticos isotrópicos, usando expressões derivadas da teoria da elasticidade linear (Timoshenko, 1951; Aki \& Richards, 1980). Assim, as constantes de Lamé são expressas da seguinte forma:

$$
\lambda=\rho\left(V_{P}^{2}-2 V_{S}^{2}\right),
$$

e

$$
\mu=\rho V_{S}^{2},
$$


onde os registros do perfis de densidades efetivas são representados por $\rho$, e as estimativas das velocidades de ondas compressionais e cisalhantes por $V_{P}$ e $V_{S}$, respectivamente. O módulo de Young E pode ser avaliado em função das estimativas das constantes de Lamé, a saber:

$$
\mathrm{E}=\mu \frac{3 \lambda+2 \mu}{\lambda+\mu}
$$

assim como a incompressibilidade,

$$
\kappa=\lambda+\frac{2}{3} \mu
$$

e a razão de Poisson,

$$
\sigma=\frac{\lambda}{2 \lambda+\mu}
$$

As relações anteriores são oriundas da teoria da elasticidade linear, e se aplicam a sólidos elásticos, homogêneos e isotrópicos. No entanto, essas equações podem ser aplicadas para avaliação de propriedades elásticas em materiais heterogêneos, como é o caso de rochas sedimentares. Assim, aplicamos as equações descritas acima usando perfis geofísicos de um poço no reservatório Namorado. Os resultados são mostrados a seguir.

\section{RESULTADOS}

Para construção dos perfis do módulo de cisalhamento $\mu$, do coeficiente de Lamé $\lambda$, do módulo de Young $\mathrm{E}$ e da razão de Poisson $\sigma$, utilizamos os perfis geofísicos do poço NA 01, cuja localização está mostrada na figura 1. Os perfis geofísicos de densidade e de velocidades sísmicas, e o perfil composto desse poço, estão exibidos no primeiro e último painéis, respectivamente, da figura 2.

$\mathrm{Na}$ interpretação dos perfis de módulos elásticos, levamos em consideração os aspectos quantitativos e qualitativos das estimativas. Para a efetuarmos a interpretação quantitativa, assumimos intervalos de variação dos módulos elásticos $\mu$ e $\kappa$ para os litotipos no poço NA 01. Segundo Schön (1996), o módulo de rigidez para arenitos está no intervalo de $7 \mathrm{GPa} \leq \mu \leq 24 \mathrm{GPa}$, a incompressibilidade $15.5 \mathrm{GPa} \leq \kappa \leq 39.5 \mathrm{GPa}$. Em folhelhos, $1.5 \mathrm{GPa} \leq \mu \leq 7.0 \mathrm{GPa}$ e $7.5 \mathrm{GPa} \leq \kappa \leq 17 \mathrm{GPa}$. Comparando o perfil composto (estratigráfico) do poço NA01 com as estimativas dos perfis de módulos elásticos, interpretamos que, entre as profundidades $3000 \mathrm{~m}$ e $3005 \mathrm{~m}$, os módulos elásticos $\mu$ e $\kappa$ do arenito produtor de óleo estão nos intervalos descritos na literatura. O decréscimo do módulo de rigidez e o módulo de compressão conduz a interpretação de uma camada de foIhelho (rocha selante), o que está em desacordo com a estratigrafia no perfil composto. A partir de $3025 \mathrm{~m}$, observamos, de acordo com o perfil composto, a intercalação de rochas selantes (margas, calcilutitos e foIhelhos) e camadas de arenitos produtores. Na profundidade de $3100 \mathrm{~m}$, também de acordo com a estratigrafia, temos calcilutito delimitando a base do reservátorio indicando aumento nos módulos de rigidez e de compressão. Todavia, entre 3130 e 3133 m observamos um

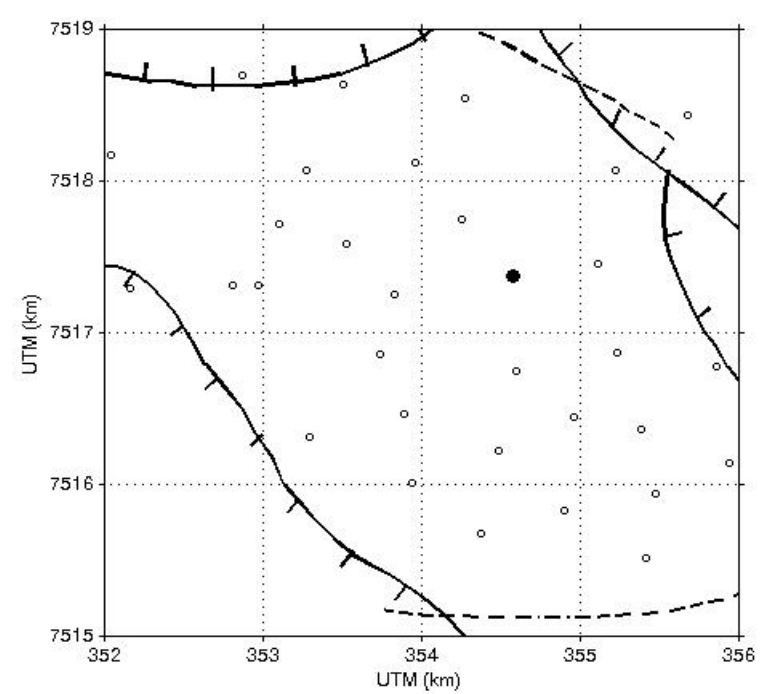

Figura 1: Localização do poço NA01 (círculo hachurado) no mapa estrutural do reservatório Namorado. Os demais círculos representam poços não utilizados nesse trabalho. A figura mostra os limites estruturais do reservatório. Modificado de Augusto (2009).

aumento dos módulos de rigidez e de compressão, indicando homogeneidade para a camada de arenito correspondente.

Na próxima etapa deste trabalho efetuaremos a interpretação qualitativa, construindo perfis de módulos elásticos para os demais poços do reservatório de Namorado. Posteriormente, construiremos mapas representativos da variação de módulos elásticos para determinadas profundidades.

\section{DISCUSSÃO E CONCLUSÕES}

A metodologia utilizada neste trabalho permite construir perfis de módulos elásticos a partir de perfis geofísicos, conduzindo à compreensão do comportamento geomecânico dos sedimentos atravessados pelos poços correspondentes. Salientamos que este trabalho é parte de uma dissertação de mestrado, que tem por objetivo a construção de mapas representativos da variação dos módulos elásticos isotrópicos no reservatório Namorado. Numa etapa futura do projeto, avaliaremos os módulos elásticos anisotrópicos (simetria VTI - vertical transverse isotropy), que também deverão ser os dados de entrada para construção de mapas de módulos elásticos anisotrópicos. Dessa forma, faremos comparações entre estes dois mapas (isotroṕico e anisotrópico) para um melhor conhecimento do comportamento geomecânico do reservatório Namorado.

\section{AGRADECIMENTOS}

Lidiane Garcia reconhece o apoio da CAPES, através de uma bolsa de pesquisa, para desenvolvimento de dissertação de mestrado no Curso de PG em Geofísica do 

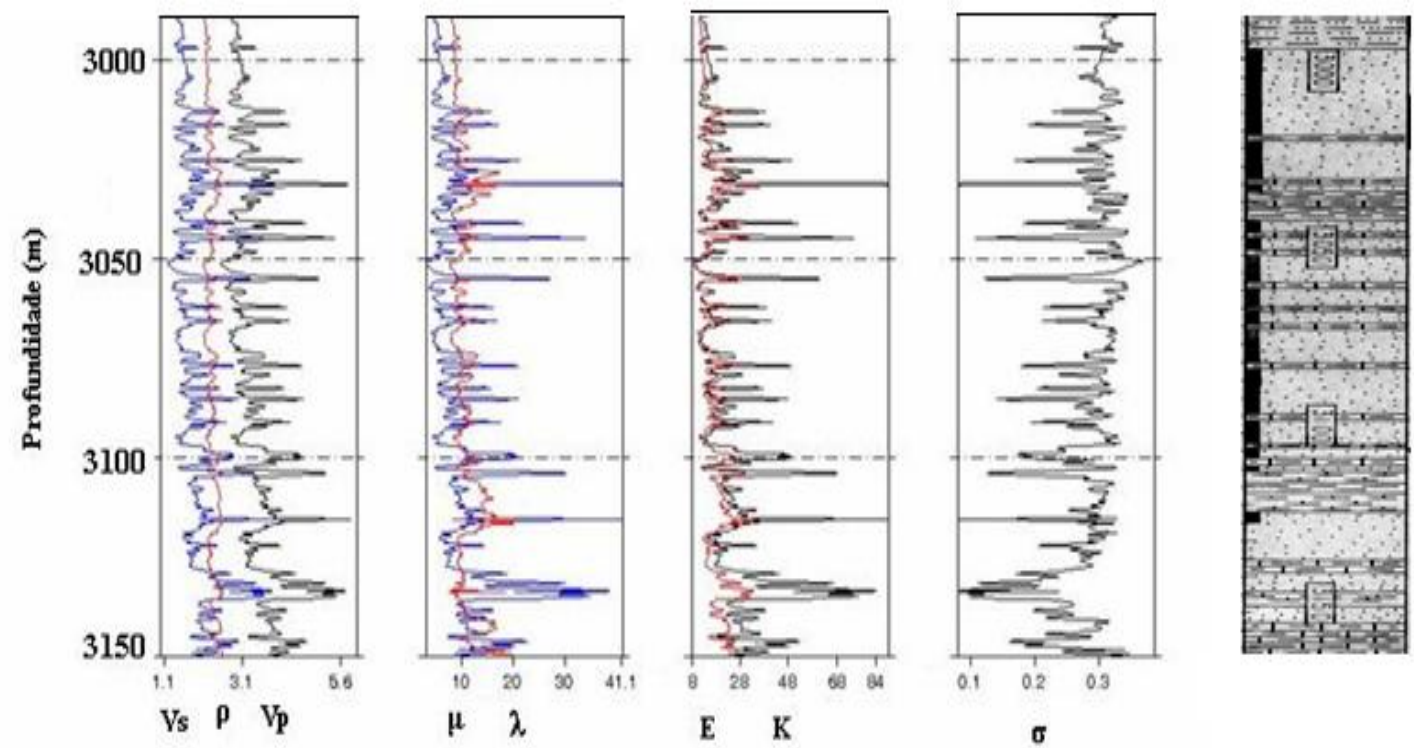

Figura 2: Perfis de módulos elásticos do poço NA01. Descrição dos perfis e código de cores: (a) Vs em azul, $\rho$ em vermelho e Vp em preto; (b) $\mu$ em azul e $\lambda$ em vermelho; (c) E em preto e $\kappa$ em vermelho; e (d) $\sigma$. O perfil litológico correspondente é mostrado à direita.

\section{ON-MCT.}

AKI, K. and RICHARDS, P. G., Quantitative Seismology - Theory and methods, Vol. I-II, 1980.

AUGUSTO, F. O. A., 2009, Mapas de Amplitude Sísmica para Incidência Normal no Reservatório Namorado, Bacia de Campos, Dissertação de Mestrado, Curso de PG em Geofísica, Observatório Nacional, MCT, 116 p.

AUGUSTO, F. O. A. e MARTINS, J. L., 2008, Relações Empíricas Não-Convencionais para Estimativa de Perfis Sônicos de Ondas Compressionais, Rio Oil \& Gas 2008 Expo and conference, Instituto Brasileiro de Petróleo, Gás e Biocombustíveis, 15-18 Setembro, Rio Centro, Rio de Janeiro, CD-ROM.

CASTAGNA, J. P., BATZLE, M. L., and EASTWOOD, R. L., 1985, Relationships between compressional-wave and shear-wave velocities in clastic silicate rocks, Geophysics, $50,571-581$.

DEWAN, J. T., 1983, Essentials of modern open-hole log interpretation, PennWell Books, $361 \mathrm{p}$.

EBERHART-PHILLIPS, D., HAN, D-H., and ZOBACK, M. D., 1989, Empirical relationships among seismic velocity, effective pressure, porosity, and clay content in sandstone: Geophysics, 54, 82-89.

ELLIS, D. V., 1987, Well logging for earth scientists, Elsevier Science Publishing Co. Inc, 550p.

HAN, D-H., NUR, A., and MORGAN, D., 1986, Effects of porosity and clay content on wave velocities in sandstones: Geophysics, 51, 2093-2107.

LEE, M. W., 2006, A simple method of predicting S-wave velocity, v.71, n.6, pp.161-164.

MILLER, S. L. M., and STEWART, R. R., 1990, Effects of lithology, porosity and shaliness on P- and Swave velocities from sonic logs: Canadian Journ. of Expl. Geophysics, 26, 94-103.

SCHÖN, J. H., 1996, Density of rocks, in: Physical properties of rocks: Fundamentals and principles of petrophysics, Chapter 3, Handbook of Geophysical Exploration, Seismic Exploration, Vol. 18, 583 p.

TIGRE, C. A., and LUCCHESI, C. F., 1986, Estado atual do desenvolvimento da Bacia de Campos e perspectivas. In: Seminário de Geologia de Desenvolvimento e Reservatório, DEPEX-PETROBRAS, Rio de Janeiro, 112.

TIMOSHENKO, S. and GOODIER, J. N., Theory of elasticity, Vol. I-II, 1951. 